\title{
The Effects of Breastfeeding on the Process of Tooth and Jaw's Development
}

\author{
Mohammed Karimi DMD, BS* \\ Department of Pediatric Dentistry, Sepideh Dental Clinic, Iran
}

*Corresponding author: Mohammed Karimi, Department of Pediatric Dentistry, Sepideh Dental Clinic, Iran

\begin{abstract}
From the nutritional point of view, it has been proven that breast milk has many benefits for the baby, and it is advisable for all mothers to give their baby milk, and if possible do not replace that with the bottle. In other words, we can say that the sucking mechanism used during bottle-feeding is markedly different from that used during breast-feeding. The Federation of Orthodontists of France announced in a report that breastfeeding not only prevents allergies and gastrointestinal infections and overweight, it also promotes the regular growth of the baby's face. Some of the researches prove this hypothesis.
\end{abstract}

Keywords: Bottle Feeding; Breast Feeding; Sucking Mechanism; Growth of Baby Face; Allergies; Gastro Intestinal Infections; Overweight

\section{Introduction}

After birth, the baby learns how to suck on her mother's breast. She instinctively brings forward her lower jaw and tongue; then starts sucking with full power so that all the muscles of her tongue, cheeks, lips, and jaw are involved. In all infants, since jaws are not fully developed at birth, sucking breast milk helps the jaw to grow as well as the teeth in the future [1-3]. Breast-feeding has been indicated as one of the main factors which are responsible for the correct growth and formation of dentofacial structures during the infancy [1-3]. Breastfeeding is a useful action for developing and growing teeth and jaws of infants [1-4]. The mechanism used in for the time of bottle-feeding is markedly different from that used during breast-feeding [5-7]. In the course of sucking mother's milk, more muscles are activated to get milk than to drink milk from the bottles. During this action, the baby inserts more of the nipple into his mouth, consequently, moves the jaw up and down, and sucks the breast with all force to release the milk. To achieve this, the facial and oral muscles of the baby are involved in milking activities. This improves the shape of the jaws, and healthy teeth are expected to be in the correct eruption direction without any deviation and abnormalities [1-4].

\section{The Main Cause of Abnormal Tooth Formation During Infancy}

One of the factors leading to abnormal teeth and also leading children to orthodontic or speech therapies [8] is the abnormal orofacial muscular imbalance pattern of the tongue [9-11] known as tongue thrust. This problem is more common among children who are fed through the bottle and is often not seen among those who are breastfed. In other words, the breast-fed baby has more forceful gums and mandibles to extract the milk from the mother's breast while a baby who is fed with a bottle, does not have to use extra jaws force because by a simple sucking a rapid flow of milk will be obtained. Of course, it should not be taken for granted that all children who use the milk bottle suffer from jaw problems, but it should be remembered that breastfeeding give better evolution to the jaws and teeth than the nourishment from the bottle.

\section{Overview of Some Researches}

The early transition from breastfeeding to bottle-feeding may contribute to inadequate mandibular development which can be a dominant and deleterious factor in the development of occolusofacial problems. In this part, we look at some research which may point out this strong hypothesis. Some studies have cited that breastfeeding is a protective factor against malocclusion: Labbok and Hendershot have suggested that increased bottlefeeding duration may contribute to the prevalence of malocclusions [12].

Viggiano et al. and Karjalainen et al. have indicated that breastfeeding can be a positive factor to prevent the development of posterior cross bite in the primary dentition $[6,13]$. Warren et al. 
reported that breastfeeding promotes normal palate development and weakens the formation of a deep and high-arched palate [14]. Several studies agree that bottle-feeding may be responsible for the development of sucking habits which may lead to some forms of malocclusion $[6,14,15]$.

\section{Conclusion}

Breastfeeding acts on the process of sucking which are influencing the development of facial bones and muscles. Infants who are breastfed have greater facial muscle activities compare to those who are bottle-fed. In other words, breast feeders present an excellent orofacial muscle work out which helps to develop good their bony jaw structures. Moreover, breastfeeding prevents against orthodontic problems and malocclusions (for instance: overbite, posterior crossbite, tongue thrust, oral habits and etc.) that are cited in some researches.

\section{References}

1. Romero CC, Scavone Junior H, Garib DG, Cotrim Ferreira FA, Ferreira RI (2011) Breastfeeding and non-nutritive sucking patterns related to the prevalence of anterior open bite in the primary dentition. J Appl Oral Sci 19(2): 161-8.

2. Degano MP, Degano RA (1993) Breastfeeding and oral health; A primer for the dental practitioner. N Y State Dent J 59(2): 30-32.

3. Sánchez Molins M, Grau Carbó J, Lischeid Gaig C, Ustrell Torrent JM (2010) Comparative study of the craniofacial growth depending on the type of lactation received. Eur J Paediatr Dent 11(2): 87-92.

4. The Infant Feeding Guidelines (2012) and the Australian Dietary Guidelines (2013), which form the basis for nutrition policy in Australia.
5. Inoue N, Sakashita R, Kamegai $\mathrm{T}$ (1995) Reduction of masseter muscle activity in bottle-fed babies. Early Hum Dev 42(3): 185-193.

6. Viggiano D, Fasano D, Monaco G, Strohmenger L (2004) Breastfeeding, bottle feeding, and non-nutritive sucking; effects on occlusion in deciduous dentition, Arch Dis Child 89(12): 1121-1123.

7. Gomes CF, Trezza EM, Murade EC, Padovani CR (2006) Surface electromyography of facial muscles during natural and artificial feeding of infants. J Pediatr (Rio J) 82(2): 103-109.

8. Proffit W, Fields HW, Sarver DM(2013) Contemporary Orthodontics: Mosby, $5^{\text {th }}$ edition pp. 768

9. Subtelny JD (1973) Oral habits studies in form, function, and therapy, The Angle Orthodontist 43(4): 347-383.

10. Sinem T, Biren S, Ceylanoglu C (2010) Tongue Pressure Changes Before, During and After Crib Appliance Therapy. Angle Orthod 80: 533-539.

11. Jann Gladys Reid (1962) Orofacial muscle imbalance, The Journal of the American Dental Association 65(6): 767-779.

12. Labbok MH, Hendershot GE (1987) Does breastfeeding protect against malocclusion? An analysis of the 1981 Child Health Supplement to the National Health Interview Survey. Am J Prev Med 3(4): 227-232.

13. Karjalainen S, Ronning O, Lapinleimu H, Simell O (1999) Association between early weaning, non-nutritive sucking habits and occlusal anomalies in 3-year-old Finnish children. Int J Paediatr Dent 9(3): 169173.

14. Warren JJ, Bishara SE, Steinbock KL, Yonezu T, Nowak AJ (2001) Effects of oral habits' duration on dental characteristics in the primary dentition. J Am Dent Assoc 132(12): 1685-1693.

15. Farsi NM, Salama FS (1997) Sucking habits in Saudi children: prevalence, contributing factors, and effects on the primary dentition, Pediatr Dent 19(1): 28-33.

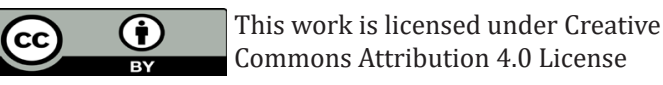

To Submit Your Article Click Here: Submit Article

DOI: $10.32474 /$ IPDOAJ.2019.03.000165

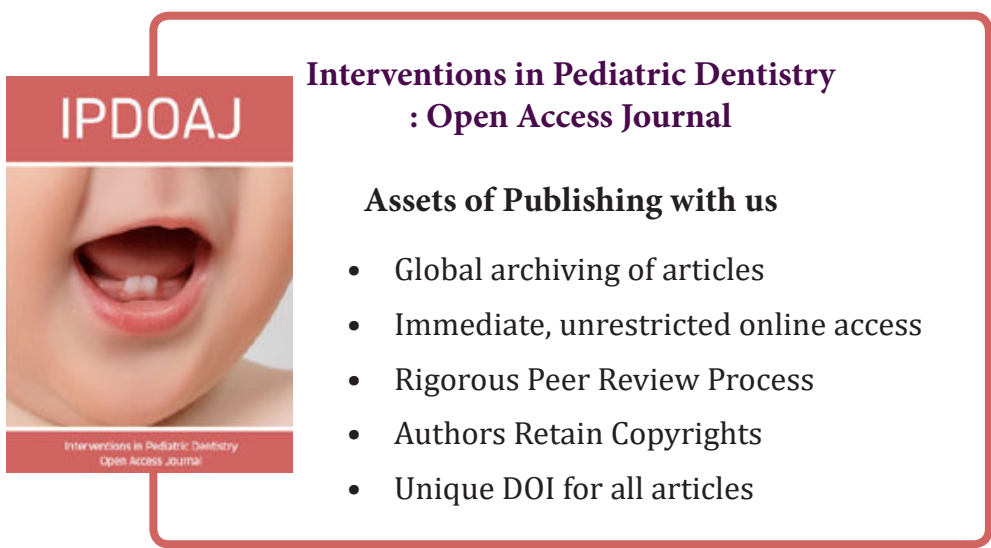

